\title{
Hippocampal train stimulation modulates recall of fear extinction independently of prefrontal cortex synaptic plasticity and lesions
}

\author{
Mélissa Farinelli, Olivier Deschaux, Sandrine Hugues, Aurélie Thevenet, \\ and René Garcia ${ }^{1}$ \\ Laboratoire de Neurobiologie et Psychopathologie, JE2441, Université de Nice-Sophia Antipolis, 06108 Nice, France
}

\begin{abstract}
It has been shown that long-term potentiation (LTP) develops in the connection between the mediodorsal thalamus (MD) and the medial prefrontal cortex (mPFC) and between the hippocampus (HPC) and the mPFC following fear extinction, and correlates with extinction retention. However, recent lesion studies have shown that combined lesions of the MD and mPFC do not interfere with extinction learning and retention, while inactivation of the dorsal HPC disrupts fear extinction memory. Here we found in rats that immediate post-training HPC low-frequency stimulation (LFS) suppressed extinction-related LTP in the HPC-mPFC pathway and induced difficulties in extinction recall. HPC tetanus, applied several hours later, failed to re-establish mPFC LTP but facilitated recall of extinction. Delayed post-training HPC LFS also provoked mPFC depotentiation and difficulties with extinction recall. HPC tetanus abolished these two effects. We also found that damage to the MPFC induced fear return only in rats that received HPC LFS following extinction training. HPC tetanus also reversed this behavioral effect of HPC LFS in lesioned rats. These data suggest that the HPC interacts with the MPFC during fear extinction, but can modulate fear extinction independently of this interaction.
\end{abstract}

Mouse studies have shown that fear extinction is associated with long-term potentiation (LTP)-like changes in the projection from the mediodorsal thalamus (MD) to the medial prefrontal cortex (mPFC) (Herry et al. 1999; Herry and Garcia 2002). Follow-up tests revealed that these MPFC LTP-like changes develop only in mice that easily recall their fear extinction (Herry and Garcia 2002). In addition, difficulties in recall of fear extinction have also been reported in pharmacological studies in which rats were infused with drugs, such as a protein synthesis blocker (Santini et al. 2004) and an inhibitor of mitogen-activated protein kinases (Hugues et al. 2004), which are known to impair LTP-related mechanisms. These findings, together with lesion studies showing that rats without the MPFC have difficulties recalling fear extinction acquired the previous day (Quirk et al. 2000; Lebron et al. 2004), also support the involvement of mPFC LTP in longterm fear extinction. However, more recent studies have shown that pre-conditioning or post-extinction lesions of the mPFC, even combined with damage to the MD, do not impair memory of fear extinction (Garcia et al. 2006), suggesting that the mPFC is not necessary for extinction of conditioned fear in all experimental conditions.

Another study from our group has revealed that extinction of conditioned fear is also associated with LTP-like changes in the projection from the ventral hippocampus (HPC) to the mPFC (S. Hugues, A. Chessel, I. Léna, R. Marsault, and R. Garcia, in prep.). As both the dorsal and ventral HPC are involved in fear extinction (Cannich et al. 2004) and inactivation of the dorsal HPC interferes with fear extinction (Corcoran et al. 2005), we hypothesized that the HPC may interact with the mPFC in relation to fear extinction, but can modulate fear extinction independently of this interaction.

\footnotetext{
'Corresponding author.

E-mail rgarcia@unice.fr; fax (33) 492-07-61-62.

Article and publication are at http://www.learnmem.org/cgi/doi/10.1101/ Im.204806.
}

To address this, we first examined whether LTP-like changes also develop in the dorsal HPC-mPFC pathway following fear extinction in rats, as found for the ventral HPC-mPFC pathway (S. Hugues, A. Chessel, I. Léna, R. Marsault, and R. Garcia, in prep.). We then determined whether HPC LFS would disrupt development of such changes and be associated with impairment of recall of extinction, and whether HPC tetanus would restore both mPFC LTP and expression of fear extinction. Finally, we analyzed whether HPC LFS and tetanus would modulate expression of fear extinction in rats with mPFC lesions.

\section{Results}

\section{Extinction-related LTP in the HPC-mPFC pathway}

Single test stimulation of the dorsal HPC (Fig. 1A) evoked in the mPFC (Fig. 1B) a field potential characterized by a monosynaptic negative peak (N peak) at a 20-msec latency (Fig. 1C), as described by others (Romcy-Pereira and Pavlides 2004). Before and after conditioning (tone-shock pairings), amplitude of this $\mathrm{N}$ peak was stable in both shocked (FC) and nonshocked (NFC) rats. In addition, the two groups did not differ electrophysiologically from each other before and after conditioning. This indicates that the application of eyelid shock (unconditioned stimulus) did not alter synaptic efficacy in the HPC-mPFC pathway of the FC rats. However, after extinction training (25 CS-alone presentations), the FC group displayed increases in the amplitude of the $\mathrm{N}$ peak that were still present up to $72 \mathrm{~h}$ later, while responses of NFC rats stayed at baseline levels during all recording sessions (Fig. $2 \mathrm{~A})$. Because the NFC rats also received the same number of CS presentations, LTP-like changes observed with the FC rats were therefore a consequence of extinction learning rather than a result of repetitive presentations of the tone CS-alone. A two-way ANOVA performed on these data showed a main effect of group $\left(F_{1,8}=48.4 ; P<0.0001\right)$ and session $\left(F_{15,120}=8.6 ; P<0.0001\right)$ and a group $\times$ session interaction $\left(F_{15,120}=8.1 ; P<0.0001\right)$. Post hoc Scheffe tests indicated that extinction-related LTP was significant immediately after training and throughout the entire 
72-h recording period (all $P$-values $<0.05$ ). The same analyses for the NFC group confirmed that mPFC response amplitudes were stable across all recording sessions.

Behavioral data (Fig. 2B) showed that freezing levels were low during all sessions of CS presentations in NFC animals. FC rats acquired fear during conditioning as revealed by high levels of freezing during the first five CS presentations (early extinction). Continuing presentations of the CS extinguished this behavior, which reached low values during the last five CS presentations (i.e., trials 21-25: late extinction), similar to those displayed by the NFC group. Seventy-two hours after this training, re-exposure to the CS (five presentations: retention test) did not evoke high levels of freezing (absence of spontaneous recovery). A two-way ANOVA performed on these data (three session levels: early extinction, late extinction, and retention test) indicated a main effect of group $\left(F_{1,8}=59.4 ; P<0.0001\right)$ and session $\left(F_{2,16}=72.8 ; P<0.0001\right)$ and a group $\times$ session interaction $\left(F_{2,16}=66.6 ; P<0.0001\right)$. Post hoc Scheffe tests indicated that the two groups did not differ from each other during both late extinction and retention test. In other words, extinction was fully recalled in the FC group.

\section{Effects of immediate post-training suppression of extinction-related mPFC LTP}

Two groups of rats (iLFS-T and iLFS-NT) with stable electrophysiological responses before and after conditioning (tone-shock pairings) were submitted to extinction (25 CS-alone presentations) of their conditioned freezing behavior and received HPC LFS immediately after this training (iLFS). HPC LFS produced decreases in the amplitude of the N peak of the HPC-mPFC field potential that were still present for both groups up to $72 \mathrm{~h}$ later. Application of HPC tetanus (iLFS-T) suppressed this long-term depression (LTD) but did not restore extinction-like potentiation in the mPFC (Fig. 3A). A two-way ANOVA performed on these data (iLFS-T) and data from rats that did not receive HPC tetanus before the retention test (iLFS-NT) revealed no main effect of group $\left(F_{1,8}<0.4\right)$. However, there was a main effect of session $\left(F_{15,120}=7.5 ; P<0.0001\right)$ and a significant session $\times$ group interaction $\left(F_{15,120}=2.3 ; P=0.01\right)$. Post hoc Scheffe tests on the
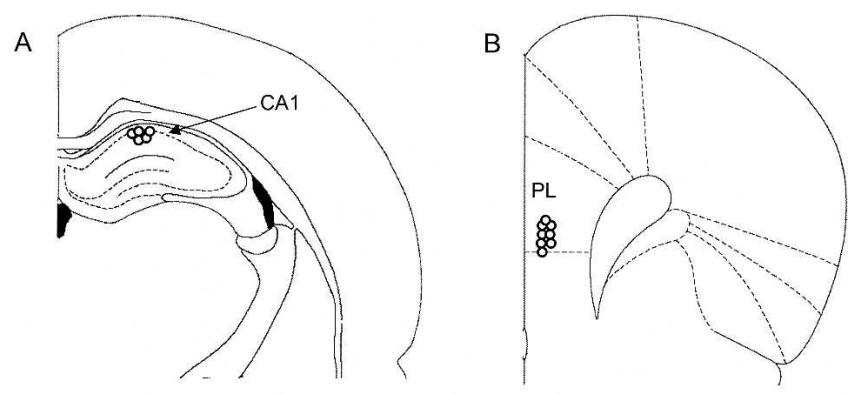

C

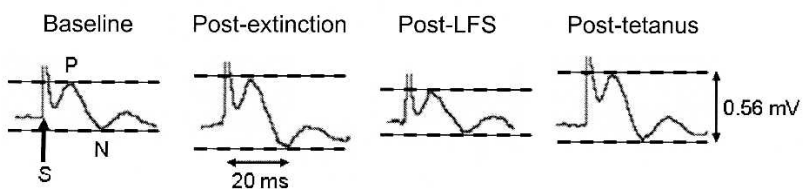

Figure 1. $(A, B)$ Diagrams showing electrode placements (open circles) in the dorsal CA1 area of the hippocampus ( $A$ : stimulation) and the ventral part of the prelimbic area (PL) of the medial prefrontal cortex ( $B$ : recording). ( $C$ ) Representative changes in field potential (from baseline) following extinction training (Post-extinction), CA1 low-frequency stimulation (Post-LFS), and tetanus (Post-tetanus). Changes in the negative peak were measured between the two dotted lines separating the first positive $(P)$ and negative $(N)$ peaks. (S) Stimulus artifact.

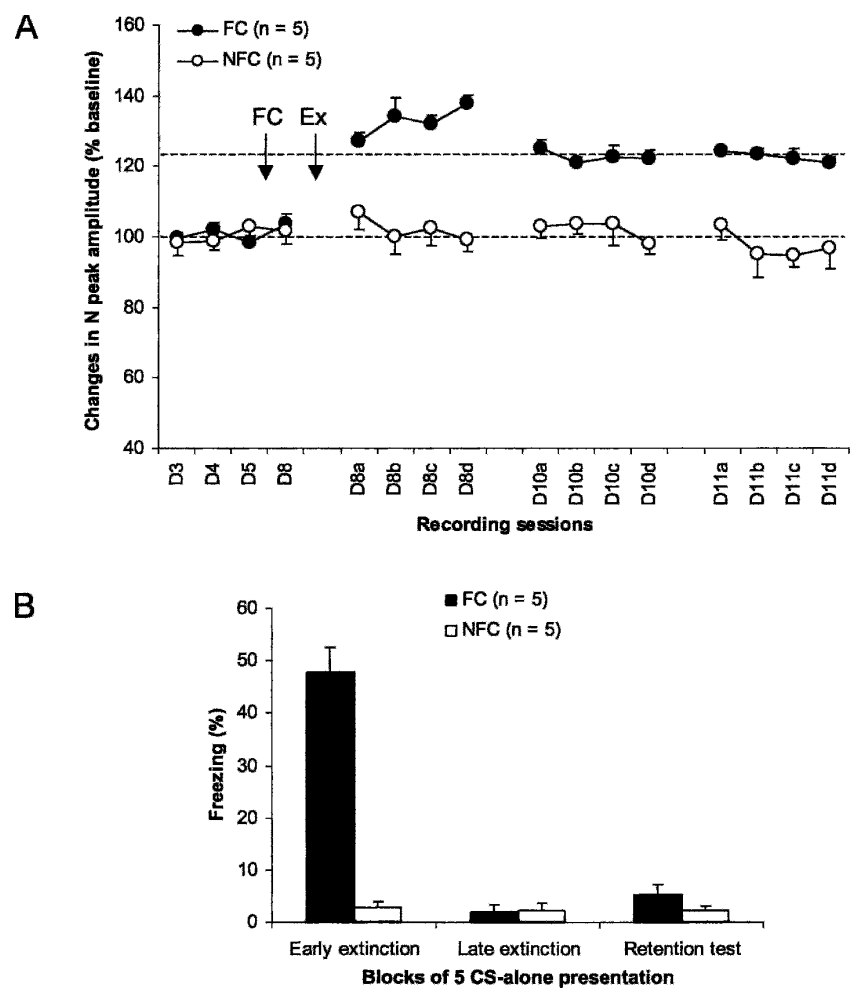

Figure 2. ( $A$ ) Changes (mean \pm SEM percent relative to baseline) in the amplitude of the negative $(\mathrm{N})$ peak of the prefrontal field potential at baseline (D3-D4: lower dotted line), after fear conditioning (FC), and after extinction training (Ex: upper dotted line). (B) Freezing behavior (mean \pm SEM) during the first (early extinction) and last (late extinction) five CS presentations of extinction training (25 CS presentations) and during the retention test (five CS presentations).

same data before HPC tetanus showed that in both groups, LTD induced by HPC LFS was significant relative to baseline values (all $P<0.05)$. After tetanus, values of $\mathrm{N}$-peak amplitude in the iLFS-T group did not differ from baseline values or from those of the iLFS-NT group.

Behavioral data showed that both groups similarly acquired (as shown in early extinction) and extinguished (as shown in late extinction) their conditioned freezing behavior to the tone CS (Fig. 3B). However, during the retention test, levels of freezing were again high in the iLFS-NT group, while freezing remained low in the iLFS-T group, indicating that immediate post-training HPC LFS blocked expression of extinction and that HPC tetanus restored this function. A two-way ANOVA performed on these data (three session levels: early extinction, late extinction, and retention test) showed a main effect of group $\left(F_{1,8}=6.1 ; P<0.05\right)$ and session $\left(F_{2,16}=98.8 ; P<0.0001\right)$ and a group $\times$ session interaction $\left(F_{2,16}=12.1 ; P<0.001\right)$. Post hoc Scheffe tests indicated that the two groups differed from each other only during the retention test $(P<0.001)$. Further analyses confirmed that freezing levels observed during the retention test in the iLFS-NT group were significantly higher than those observed during late extinction $(P<0.001)$ and did not differ from those observed during early extinction. In contrast, freezing levels recorded during the retention test in rats that received tetanus (iLFS-T group) differed only from those observed during early extinction $(P<0.0001)$.

\section{Effects of delayed post-training suppression of extinction-related mPFC LTP}

Two groups of rats (dLFS-T and dLFS-NT) with stable response amplitudes before and after fear conditioning (tone-shock pair-

\section{Learning \& Memory}

www.learnmem.org 
A

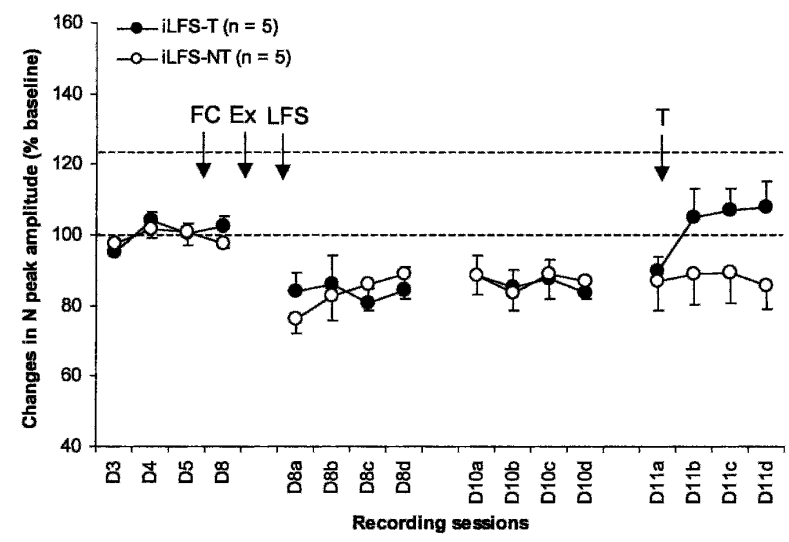

B

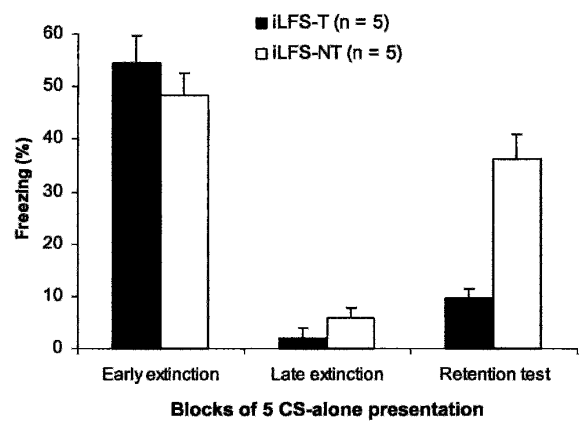

Figure 3. Effects of immediate post-training low-frequency stimulation (LFS) and pre-retention test tetanus (T) of the hippocampus. (A) Changes (mean \pm SEM percent relative to baseline) in the amplitude of the negative $(\mathrm{N})$ peak of the prefrontal cortex field potential at baseline (D3-D4: lower dotted line), after fear conditioning (FC), and post-extinction (Ex) hippocampal LFS and tetanus. Hippocampal tetanus failed to restore extinction-related potentiation (upper dotted line). (B) Freezing behavior (mean \pm SEM) during the first (early extinction) and last (late extinction) five CS presentations of extinction training (25 CS presentations) and during the retention test (five CS presentations) in rats that received both LFS and tetanus (iLFS-T) and in rats that received only LFS (iLFS-NT).

ings) were re-exposed to the tone CS, and HPC-mPFC field potentials were recorded during the $2 \mathrm{~h}$ that followed extinction training and $48 \mathrm{~h}$ later (Fig. 4A). Both groups displayed increases in the amplitude of the N peak of HPC-mPFC field potential that were still present at the 48 -h recording session. At this posttraining delay, HPC LFS (dLFS) induced depotentiation to baseline-like values that was still present $24 \mathrm{~h}$ later. Application of HPC tetanus (dLFS-T) restored the pre-LFS values of LTP (Fig. 4A). A two-way ANOVA performed on data from rats with and without HPC tetanus (dLFS-T and dLFS-NT, respectively) did not indicate any main effect of group $\left(F_{1,8}<0.3\right)$, while a main effect of session and a significant session $\times$ group interaction were found $\left(F_{15,120}=18.2 ; P<0.0001\right.$, and $F_{1,120}=1.8 ; P<0.05$, respectively). Post hoc Scheffe tests on data before HPC LFS showed significant extinction-related LTP immediately after extinction training and $48 \mathrm{~h}$ later in both groups (all $P$-values $<0.05$ ). After LFS, values of N-peak amplitude did not differ from baseline levels for either group. However, after HPC tetanus, values of N-peak amplitude were again different from baseline levels (six levels; $P<0.05$ ); these values did not differ from extinction-related LTP levels, but differed from those of rats that did not receive HPC tetanus (all $P$-values $<0.05$ ).

Behavioral data showed again that both groups acquired (as shown in early extinction) and extinguished (as shown in late extinction) their conditioned freezing behavior to the tone CS (Fig. 4B). However, during the retention test, levels of freezing returned to early extinction-like values in the dLFS-NT group, while levels of freezing remained at late extinction-like values in the dLFS-T group, indicating that delayed post-training HPC LFS also blocked the expression of extinction and that tetanus restored extinction. A two-way ANOVA performed on these data (three session levels: early extinction, late extinction, and retention test) showed a main effect of group $\left(F_{1,8}=8.9 ; P<0.05\right)$ and session $\left(F_{2,16}=39.1 ; P<0.0001\right)$ and a group $\times$ session interaction $\left(F_{2,16}=4.9 ; P<0.05\right)$. Post hoc Scheffe tests indicated that the two groups differed from each other only during the retention test $(P<0.0001)$. Further analyses confirmed that freezing levels observed during the retention test in the dLFS-NT group were significantly higher than those recorded during late extinction $(P<0.01)$ and did not differ from those expressed during early extinction. In contrast, freezing levels in the dLFS-T group differed only from those recorded during early extinction $(P<0.0001)$.

\section{HPC modulation of extinction memory in rats} with $\mathrm{mPFC}$ lesions

Three groups of rats with mPFC lesions (mPFC, mPFC + LFS, and $\mathrm{mPFC}+\mathrm{LFS}+\mathrm{T}$ groups) (Fig. 5A) and sham-operated rats (Sham group) were submitted to fear conditioning (tone-shock pairings) and extinction of their conditioned freezing behavior to the tone. All groups acquired (as shown in early extinction) and extinguished (as shown in late extinction) their conditioned freez-

A

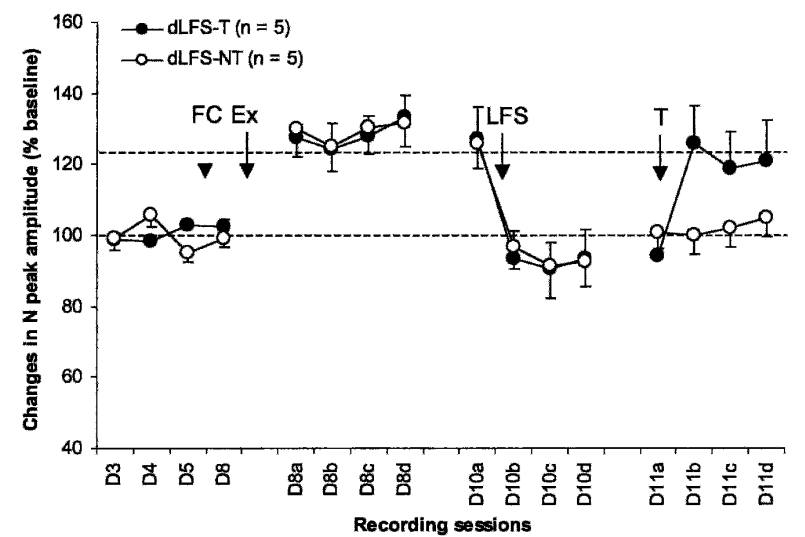

B

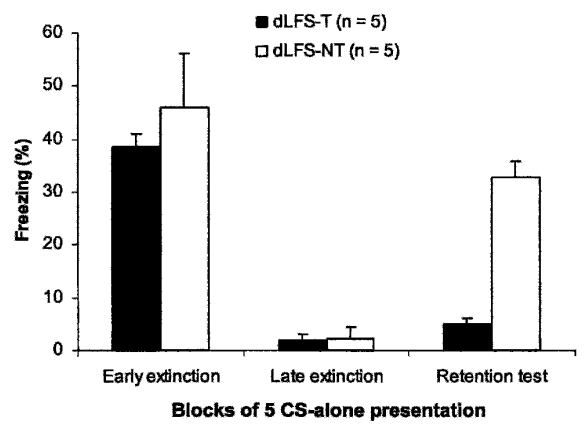

Figure 4. Effects of delayed post-training low-frequency stimulation (LFS) and pre-retention test tetanus ( $T$ ) of the hippocampus. ( $A$ ) Changes (mean \pm SEM percent relative to baseline) in the amplitude of the negative $(\mathrm{N})$ peak of the prefrontal field potential at baseline (D3-D4: lower dotted line) and after fear conditioning (FC), extinction (Ex), and different hippocampal train stimulations. Hippocampal tetanus restored extinction-related potentiation (upper dotted line). (B) Freezing behavior (mean \pm SEM) during the first (early extinction) and last (late extinction) five CS presentations of extinction training (25 CS presentations) and during the retention test (five CS presentations) in rats that received both LFS and tetanus (dLFS-T) and in those that received only LFS (dLFS-NT). 
A

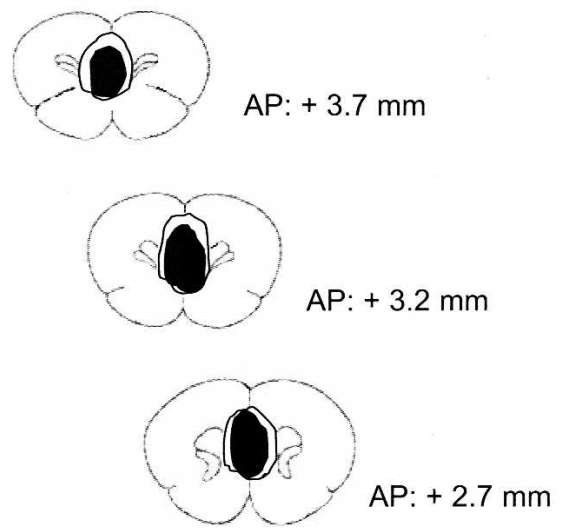

B

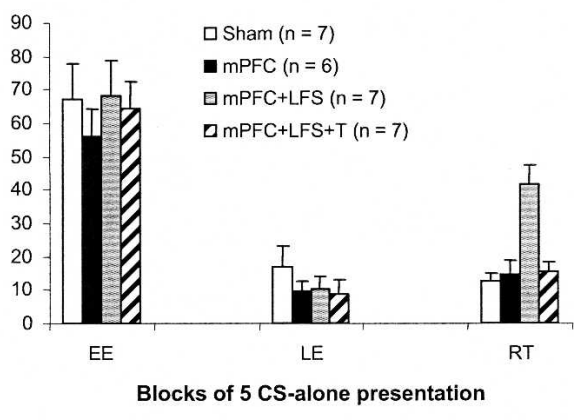

Figure 5. (A) Histology: schematic representation of brain lesions mapped onto coronal rat brain sections showing the extent of the largest (outline) and smallest (filled) electrolytic lesions in the medial prefrontal cortex. Note that lesions included both prefrontal prelimbic and infralimbic areas. Each group of lesioned rats initially comprised seven animals. (B) Freezing behavior (mean \pm SEM) during the first (early extinction: EE) and last (late extinction: LE) five CS presentations of extinction training (25 CS presentations) and during the retention test (RT; five CS presentations) in all groups.

ing behavior (Fig. 5B: EE and LE, respectively). However, during the retention test (Fig. 5B: RT), levels of freezing behavior were high in lesioned rats that received immediate HPC LFS after extinction $(\mathrm{mPFC}+\mathrm{LFS})$, but not in rats that received both immediate post-training HPC LFS and HPC tetanus before the retention test $(\mathrm{mPFC}+\mathrm{LFS}+\mathrm{T})$. Levels of freezing remained low in the Sham group and in lesioned rats that did not receive any HPC train stimulation (mPFC group). A two-way ANOVA performed on the extinction data (two session levels: early and late extinction) showed that the four groups extinguished similarly (no group effect: $F_{3,23}<1$; main effect of session: $F_{1,23}=123$; $P<0.0001)$. Direct between-group comparisons revealed that the $\mathrm{mPFC}+\mathrm{LFS}$ group differed from each of the other groups during the retention test (Scheffe test: all $P$-values $<0.05$ ). The three other groups (i.e., Sham, mPFC, and $\mathrm{mPFC}+\mathrm{LFS}+\mathrm{T}$ ) did not differ from each other. Moreover, the mPFC + LFS group exhibited significantly higher levels of freezing during the retention test than during late extinction $(P<0.05)$, indicating fear recovery for this group.

\section{Discussion}

Our results show that fear extinction is accompanied by longlasting increases in synaptic efficacy (LTP-like synaptic changes) in the projection from the dorsal HPC to the mPFC. When HPC LFS was given immediately or $48 \mathrm{~h}$ after extinction training, it suppressed this extinction-related synaptic plasticity. In both cases (immediate and delayed post-training LFS), recall of extinction was impaired. HPC tetanus, given several hours later, failed to restore mPFC LTP in the immediate post-training LFS group, but facilitated recall of extinction in both cases (immediate and delayed post-training LFS groups), indicating the possibility of protection against fear return in certain circumstances (here HPC LFS) even in the absence of mPFC LTP. In addition, lesions of the mPFC did not interfere with the behavioral effects of HPC LFS and tetanus, supporting the idea of an involvement of other HPC outputs in the modulation of long-term memory of fear extinction.

Extinction-related LTP in the MPFC was first reported in mice. It was found that the monosynaptic component of MDmPFC field potentials displays LTP-like changes following extinction training (Herry et al. 1999). Potentiation of synaptic efficacy sions do not alter retention of fear extinction (Garcia et al. 2006), while inactivation of the dorsal HPC impairs fear extinction (Corcoran and Maren, 2001, 2004; Corcoran et al. 2005).

Moreover, it is possible that impairment of recall of fear extinction provoked by HPC LFS does not result from suppression of extinction-associated mPFC LTP. Indeed, we found that HPC tetanus restored extinction-like levels of mPFC LTP only when post-training depotentiation was induced several hours after training and not when it was induced immediately. However, in both cases (with or without mPFC LTP), HPC tetanus facilitated recall of extinction. In other words, HPC train stimulation can modulate memory of fear extinction independently of levels of synaptic efficacy in the HPC-mPFC pathway. This idea is also supported by our findings showing that the HPC similarly modulates fear extinction in rats with or without lesions of the mPFC area that displayed extinction-related LTP. These data are in accord with recent findings demonstrating that damage to the mPFC (including both infralimbic and prelimbic areas) does not interfere with retention of fear extinction (Garcia et al. 2006), suggesting that the mPFC is not necessary for retention or expression of extinction of conditioned fear. However, another possible explanation for the lack of effect of permanent lesions is recovery of $\mathrm{mPFC}$ function by other structures. Because the HPC projects directly to the amygdala (Maren and Fanselow 1995; Pitkanen et al. 2000), a key structure in fear extinction (Davis et al. 2003), and because electrical stimulation of the HPC has been shown to induce synaptic plasticity in the amygdala (Maren and Fanselow 1995), it is possible that HPC train stimulation caused modulation of fear extinction via changes in synaptic efficacy in the HPC-amygdala circuits. This idea is supported by numerous findings showing that extinction of conditioned fear involves synaptic plasticity-related activities (such as gene expression, protein synthesis, $\mathrm{N}$-methyl-D-aspartate receptor binding, and signaling pathway activation) in the hippocampus and amygdala (Vianna et al. 2001, 2003; Szapiro et al. 2003; Cammarota et al. 2005).

In conclusion, it seems that the mPFC is involved in fear extinction (Herry and Garcia 2002; Milad and Quirk 2002; Barrett et al. 2003; Herry and Mons 2004; Hugues et al. 2004; Santini et al. 2004) but is not necessary for this memory process (Garcia et al. 2006; this study). The HPC may interact with mPFC during processes related to long-term fear extinction memory (as shown

\section{Learning \& Memory}

www.learnmem.org 
by extinction-related LTP in the HPC-mPFC pathway), but the HPC may also modulate fear extinction independently of this interaction (as shown by both electrophysiological and lesion data).

\section{Materials and Methods}

\section{Surgery}

The experiments were performed with male Wistar rats (Dépré) in accordance with the European Community Guidelines on the care and use of laboratory animals (86/609/EEC). Five to seven days after arrival, each rat (280-350 g) was anesthetized with sodium pentobarbital $(65 \mathrm{mg} / \mathrm{kg}$, i.p.), placed in a stereotaxic frame, and ipsilaterally implanted with a pair of twisted silver electrodes (90 $\mathrm{\mu m}$ diameter; insulated except at section) for $\mathrm{mPFC}$ field potential recording in the right hemisphere or bilaterally implanted with the same type of electrodes for mPFC lesions with additional electrodes for HPC stimulation. The recording (or lesioning) and stimulating electrodes targeted the ventral part of the prelimbic area of the mPFC (AP: $3.2 \mathrm{~mm}$ anterior to bregma; ML: $0.6 \mathrm{~mm}$; DV: $3-4 \mathrm{~mm}$ from dura) and the CA1 region of the dorsal HPC (AP: $3.2 \mathrm{~mm}$ posterior to bregma; ML: $1.6 \mathrm{~mm}$; DV: 2.2 from dura), respectively. For rats in mPFC damage groups, lesions were made with anodal, constant direct current on each side $(1.2 \mathrm{~mA}$ for $12 \mathrm{sec})$; lesion electrodes were also placed in the mPFC of sham-operated rats, but no current was applied. For electrophysiological purposes, one of three screws on the skull served as the recording ground. In addition to electrode placement in the brain, two stimulating silver electrodes (110 $\mu \mathrm{m}$ diameter; insulated except at the tip with $0.4-0.6 \mathrm{~mm}$ of exposition) were inserted in the right eyelid (without altering the eyelid function) for shock administration during fear conditioning. The entire miniature system was fixed in place onto the skull with dental cement.

\section{Stimulating and recording in behaving rats}

Electrophysiological activity was recorded through a junction field effect transistor (JFET) operational amplifier connected to the headstage. Cables from the JFET were relayed at the top of the box by a multichannel rotating connector, allowing the animal free movement inside the recording chamber. Field potentials evoked in the mPFC by single-pulse stimulation $(0.1-\mathrm{msec}$ rectangular monophasic pulses) applied to the dorsal HPC were sent to an amplifier (gain $1000 \times$; bandpass $0.001-1 \mathrm{kHz}$ ) and recorded (Spike2 software; Cambridge Electronic Design) for offline analysis. Before the first baseline recording session, responses were measured as a function of stimulus strength (input-output curves: $100-1000 \mu \mathrm{A})$. An intensity corresponding to $60 \%-70 \%$ of the saturation level was chosen for the test stimulus, which was applied every 5 sec during each recording session for a total of seven field potentials.

\section{Procedure}

After 4-7 d of recovery from surgery, rats were habituated, over a 2-d period (D1-2), to being transported from the animal house to the experimental room, and to being connected to stimulating and recording cables. Electrophysiological studies started after this period. Baseline recording sessions ( 1 session/day; 3 d: D3-5) took place in a square cage (cage $\mathrm{A}, 30 \mathrm{~cm}$ side, plastic floor) that was washed with a solution containing a mixture of ethanol (50\%) and lemon scent before introducing each animal. The cage was placed inside a sound-attenuating and temperatureregulated chamber (Imetronic). Following the third baseline recording session (D5), animals were divided into three pairs of groups with five subjects per group: FC and NFC (fear conditioning and no fear conditioning, respectively), iLFS-T and iLFS-NT (immediate LFS followed by tetanus or no tetanus, respectively), and dLFS-T and dLFS-NT (delayed LFS followed by tetanus or no tetanus, respectively). Each rat was then placed in a cylindrical cage (cage B, $25 \mathrm{~cm}$ diameter, floor covered with animal bedding that was changed before introducing each animal). Cage B was also placed inside the sound-attenuating and temperatureregulated chamber and was washed with ethanol (70\%). Four minutes after being placed in cage $B$, all rats, except those in the NFC group, were subjected to five pairings of tone $(32 \mathrm{sec}, 2 \mathrm{kHz}$, $70 \mathrm{~dB}$ ) and shock (a train of eight 3.5-mA pulses at $5 \mathrm{~Hz}$; intertrial interval: $60-180 \mathrm{sec}$ ). Rats in the NFC group were exposed to five tone-alone presentations. Two minutes after the onset of the fifth tone presentation, rats were placed back in their home cages in the animal room, where they were left undisturbed for $72 \mathrm{~h}$. After this delay (D8), rats were reintroduced in cage A, where they were exposed to 25 tone-alone presentations (intertrial interval: 60 $180 \mathrm{sec}$ ). After the last tone presentation, animals of the iLFS groups were immediately subjected to HPC LFS, a train of $2 \mathrm{~Hz}$ for 25 min. Seventy-two hours later (D11), only rats in the iLFS-T group received HPC tetanus $(100 \mathrm{~Hz}, 1 \mathrm{sec})$, which was applied 75 min before the retention test, while the other rats (iLFS-NT group) served as controls for the behavioral effects of LFS. The 2-Hz-25-min parameter was defined from our preliminary studies showing that it induced either LTD when applied from baseline levels or depotentiation when applied from LTP levels in non-fear-conditioned rats. Likewise, the $100-\mathrm{Hz}-1$-sec parameter was chosen because it produced LTP in non-fear-conditioned rats. Forty-eight hours after extinction training (D10), rats in the dLFS groups also were subjected to HPC LFS. Only one group (dLFS-T) received HPC tetanus before the retention test. The other rats (dLFS-NT group) served as controls for the behavioral effects of LFS. Rats from the FC and NFC groups did not receive any HPC train stimulation (no LFS and no tetanus) after extinction training. The retention test for all animals took place in cage A, $72 \mathrm{~h}$ after extinction training (D11). In addition to baseline recording (D3-D5), field potentials were recorded before extinction training (D8), during the $2 \mathrm{~h}$ that followed extinction training (D8a-d), before (D10a), and after (D10b-d) LFS, and before (D11a) and after (D11b-d) tetanus. Rats with mPFC lesions were subjected to a regimen similar to the FC group (mPFC group), the iLFS-NT group (mPFC + LFS group), and the iLFS-T group $(\mathrm{mPFC}+\mathrm{LFS}+\mathrm{T}$ group), but without any mPFC field potential recording. Sham-operated rats (Sham group) were treated similarly to the FC group, but without mPFC field potential recordings. The behavior of each animal was monitored continuously and, when necessary, recorded via a camera-VCR-monitor system. Conditioned fear was assessed by measuring freezing behavior, defined as the absence of all movement except those associated with respiratory (Blanchard and Blanchard 1968). Freezing was scored using a time-sampling procedure. The amount of time spent freezing was measured during the 32 -sec period of each CS presentation (conditioning, extinction, and retention test).

\section{Histology and statistical analyses}

Upon completion of the experiments, rats were placed under deep anesthesia, and the tips of electrode placements in the $\mathrm{mPFC}$ and/or the dHPC were marked by passing a $0.3-\mathrm{mA}$ to $0.5-\mathrm{mA}$ current for $20 \mathrm{sec}$. The placement of the electrodes and mPFC lesions were then verified by standard histological analyses.

All data were expressed as means and standard error of the means (SEM) and analyzed by analysis of variance (ANOVA).

\section{Acknowledgments}

We thank K.M. Myers for critical reading of the manuscript. This study was supported by the University of Nice-Sophia Antipolis and a grant from the Délégation Générale pour l'Armement to S.H.

\section{References}

Barrett, D., Shumake, J., Jones, D., and Gonzalez-Lima, F. 2003.

Metabolic mapping of mouse brain activity after extinction of a conditioned emotional response. J. Neurosci. 23: 5740-5749.

Blanchard, R.J. and Blanchard, C. 1968. Crouching as an index of fear. J. Comp. Physiol. Psychol. 6: 370-375.

Cammarota, M., Bevilaqua, L.R., Barros, D.M., Vianna, M.R., Izquierdo, 
L.A., Medina, J.H., and Izquierdo, I. 2005. Retrieval and the extinction of memory. Cell. Mol. Neurobiol. 25: 465-474.

Cannich, A., Wotjak, C.T., Kamprath, K., Hermann, H., Lutz, B., and Marsicano, G. 2004. CB1 cannabinoid receptors modulate kinase and phosphatase activity during extinction of conditioned fear in mice. Learn. Mem. 11: 625-632.

Corcoran, K.A. and Maren, S. 2001. Hippocampal inactivation disrupts contextual retrieval of fear memory after extinction. J. Neurosci. 21: $1720-1726$.

- 2004. Factors regulating the effects of hippocampal inactivation on renewal of conditional fear after extinction. Learn. Mem. 11: $598-603$.

Corcoran, K.A., Desmond, T.J., Frey, K.A., and Maren, S. 2005. Hippocampal inactivation disrupts the acquisition and contextual encoding of fear extinction. J. Neurosci. 25: 8978-8987.

Davis, M., Walker, D.L., and Myers, K.M. 2003. Role of the amygdala in fear extinction measured with potentiated startle. Ann. NY Acad. Sci. 985: $218-232$.

Garcia, R., Chang, C.H., and Maren, S. 2006. Electrolytic lesions of the medial prefrontal cortex do not interfere with long-term memory of extinction of conditioned fear. Learn. Mem. 13: 14-17.

Herry, C. and Garcia, R. 2002. Prefrontal cortex long-term potentiation, but not long-term depression, is associated with the maintenance of extinction of learned fear in mice. J. Neurosci. 22: 577-583.

Herry, C. and Mons, N. 2004. Resistance to extinction is associated with impaired immediate early gene induction in medial prefrontal cortex and amygdala. Eur. J. Neurosci. 20: 781-790.

Herry, C., Vouimba, R.M., and Garcia, R. 1999. Plasticity in the mediodorsal thalamo-prefrontal cortical transmission in behaving mice. J. Neurophysiol. 82: 2827-2832.

Hugues, S., Deschaux, O., and Garcia, R. 2004. Postextinction infusion of a mitogen-activated protein kinase inhibitor into the medial prefrontal cortex impairs memory of the extinction of conditioned fear. Learn. Mem. 11: 540-543.

Lebron, K., Milad, M.R., and Quirk, G.J. 2004. Delayed recall of fear extinction in rats with lesions of ventral medial prefrontal cortex.
Learn. Mem. 11: 544-548.

Maren, S. and Fanselow, M.S. 1995. Synaptic plasticity in the basolateral amygdala induced by hippocampal formation stimulation in vivo. $J$. Neurosci. 15: 7548-7564.

Milad, M.R. and Quirk, G.J. 2002. Neurons in medial prefrontal cortex signal memory for fear extinction. Nature 420: 70-74.

Paxinos, G. and Watson, C. 1986. The rat brain in stereotaxic coordinates. Academic Press, New York.

Pitkanen, A., Pikkarainen, M., Nurminen, N., and Ylinen, A. 2000. Reciprocal connections between the amygdala and the hippocampal formation, perirhinal cortex, and postrhinal cortex in rat. A review. Ann. NY Acad. Sci. 911: 369-391.

Quirk, G.J., Russo, G.K., Barron, J.L., and Lebron, K. 2000. The role of ventromedial prefrontal cortex in the recovery of extinguished fear. J. Neurosci. 20: 6225-6231.

Romcy-Pereira, R. and Pavlides, C. 2004. Distinct modulatory effects of sleep on the maintenance of hippocampal and medial prefrontal cortex LTP. Eur. J. Neurosci. 20: 3453-3462.

Santini, E., Ge, H., Ren, K., Pena de Ortiz, S., and Quirk, G.J. 2004. Consolidation of fear extinction requires protein synthesis in the medial prefrontal cortex. J. Neurosci. 24: 5704-5710.

Szapiro, G., Vianna, M.R., McGaugh, J.L., Medina, J.H., and Izquierdo, I 2003. The role of NMDA glutamate receptors, PKA, MAPK, and CAMKII in the hippocampus in extinction of conditioned fear. Hippocampus 13: 53-58.

Vianna, M.R., Szapiro, G., McGaugh, J.L., Medina, J.H., and Izquierdo, I. 2001. Retrieval of memory for fear-motivated training initiates extinction requiring protein synthesis in the rat hippocampus. Proc. Natl. Acad. Sci. 98: 12251-12254.

Vianna, M.R., Igaz, L.M., Coitinho, A.S., Medina, J.H., and Izquierdo, I. 2003. Memory extinction requires gene expression in rat hippocampus. Neurobiol. Learn. Mem. 79: 199-203.

Received December 8, 2005; accepted in revised form February 22, 2006.

\section{Learning \& Memory}




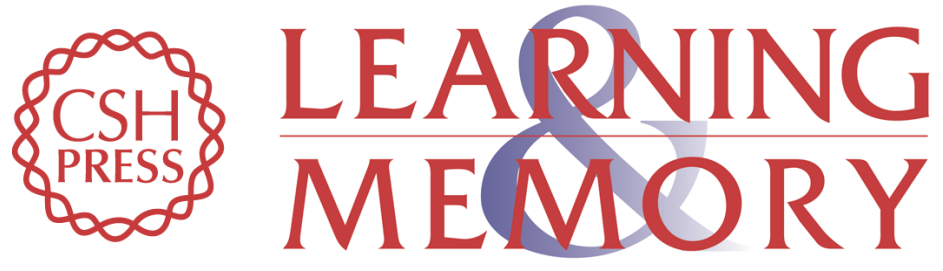

\section{Hippocampal train stimulation modulates recallof fear extinction independently of prefrontalcortex synaptic plasticity and lesions}

Mélissa Farinelli, Olivier Deschaux, Sandrine Hugues, et al.

Learn. Mem. 2006, 13:

Access the most recent version at doi:10.1101//m.204806

References This article cites 22 articles, 13 of which can be accessed free at:

http://learnmem.cshlp.org/content/13/3/329.full.html\#ref-list-1

License

Email Alerting Receive free email alerts when new articles cite this article - sign up in the box at the Service top right corner of the article or click here. 